\title{
The Extended ADS/CFT Correspondence
}

\author{
M. D. Maia \\ Instituto de Física, Universidade de Brasília, Campus da UnB, Asa Norte, 70919-970, Brasília, D.F., Brazil
}

(Received on 13 October, 2005)

\begin{abstract}
The correspondence between conformal covariant fields in Minkowski's space-time and isometric fields in the five dimensional anti-deSitter space-time is extended to a six-dimensional bulk space and its regular submanifolds, so as to include the analysis of evaporating Schwarzschild's black holes without loss of quantum unitarity.
\end{abstract}

\section{INTRODUCTION}

In 1975 S. Hawking presented his well known information loss theorem based on the semiclassical Einstein's equations:

$$
R_{\mu v}-\frac{1}{R} g_{\mu v}=8 \pi G<T_{\mu \nu}>
$$

as applied to the ordinary Kerr and Schwarzschild black holes in four-dimensional space-times. Three possible outcomes were predicted: Either the black hole evaporates leaving no trace of the properties of particles falling inside it or, after the evaporation there is a naked singularity or, lastly, there would be a regular remnant of the black hole. This result motivated a 30 year debate about the validity of the quantum unitarity near a black hole, leading to the conclusion that either something was missing in the theorem or that quantum theory in the regime of strong gravity should be modified [1].

In 2004 Hawking presented a new version of the theorem with new and different hypothesis: Now quantum gravity is approached by Euclidean Path Integral followed by a Wick rotation. Instead of the usual black holes the theorem refers to extremal black holes in the $A d S_{5}$ bulk. Finally it makes use of the ADS/CFT correspondence. The conclusion is also different: The quantum unitarity is preserved near the extremal black hole and some information can be recovered [2].

The use of different assumptions makes it difficult to compare the two theorems on equal footing. If in 1975 ordinary Special Relativity with the Poincaré symmetry was used, now the conformal symmetry of Minkowski's space is used, leaving the impression is that we are playing a different game. Nonetheless, when discussing the subject in classrooms we can hardly avoid questions such as: but then, what happened with the good old Schwarzschild's solution? Are the Schwarzschild black holes still around? If so, can we still apply the ADS/CFT correspondence? The purpose of this talk is to show that it is possible to extend that correspondence in such way that the quantum unitarity near a Schwarzschild black hole can also be ensured.

\section{CONFORMAL SYMMETRY}

Back in 1909, just after the definition of Minkowski's space-time $M_{4}$ whose metric is invariant under the Poincaré symmetry, it was found that electrodynamics was also covariant under a larger symmetry, the conformal group defined by Minkowski's metric [3]. As it was later shown by I. Segal, this is in fact the most general symmetry admitted by Maxwell's equations [4]. Therefore, following the same reasoning of Minkowski, we may ask if there would be a new Conformal Special Relativity, with a new space-time such that its metric is invariant under the conformal group of $M_{4}$.

Even before attempting an answer, it was found that the use of conformal symmetry in electrodynamics was hampered by the causality principle. For example, to maintain the conformal covariance the solutions of the electromagnetic potential wave equations must include the advanced $A_{\mu}(x+v t)$ and retarded $A_{\mu}(x-v t)$ components together. The presence of the advanced component implies in a violation of the causality principle, which was then and still is today a principle based on a solid intuition: The past must be divided from the future by the present. A denial of these facts would be a denial of our most primitive intuitions about time-order [5].

On the other hand, the discovery of the four-dimensional anti-deSitter $\left(A d S_{4}\right)$ solutions of Einstein's equations in 1917 has shown that causality may also be violated by gravity, essentially because the anti-deSitter solution admits closed time-like geodesics. Thus, if Einstein once gave us a physical meaning to the Riemann Geometry of abstract manifolds, deSitter demonstrated that such meaning is not necessarily intuitive.

In spite of this non causal implication, it was found much later that the Lie algebra of the anti-deSitter group is more consistent with the super-symmetry than the Poincare group [6]. This means that at least in some theoretical situations we may be induced to trade causality violations by some elaborated formal development, even if that theory has not yet been proven experimentally. A powerful example of this is given by the realization of type II string theory with $E_{8} \times E_{8}$ in the space $A d S_{5} \times S^{5}$ [7].

The interest in conformal symmetry reappeared in 1967, when $\mathrm{R}$. Penrose pointed out that the semi-direct product structure of the Poincare group: $P_{4}=S O(3,1) \subseteq T_{4}$, implies that the non-homogeneous action of the translation subgroup $T_{4}$ has to be handled separately from the homogeneous subgroup in the spinor representations of $P_{4}$. He suggested that the use of the conformal group leads to a more consistent spinor representation [8]. The resulting spinor structure, called twistors, is well defined in Minkowski's space-time $M_{4}$, but it still resists the generalization to curved space-times, solutions of Einstein's equations.

It should be recalled that a stronger objection to the translational subgroup $T_{4}$ existed at that time, stating that in any attempt to combine the Poincaré group with an internal or gauge 
symmetry, all particles belonging to the same spin multiplet would necessarily have zero squared mass differences. This is obviously against the experimental evidences. After much debate it was found that the problem lies within the subgroup $T_{4}$, which acts nilpotently over the remaining of the combined symmetry $[10,11]$. As we know today, the adopted solution of such No-Go problem as it become to be known, resides in the hypothesis that the combined symmetry is spontaneously broken by the Higgs mechanism. Nonetheless, in the present context, it is relevant to stress that if the Poincare symmetry is replaced by the deSitter or the anti-deSitter groups, or in fact by any semi-simple group, then the No-Go problem would be solved exactly, without supersymmetry, giving an additional support to Penrose's argument in favor of the conformal symmetry $[12,13]$.

The next step in the development of the conformal symmetry in relativist physics was the invention of the ADS/CFT correspondence in 1998 [14]. This is in essence a relation between conform-invariant fields in $M_{4}$ and the five-dimensional anti-deSitter gravitational field $A d S_{5}$ [15]. Although the correspondence was established in the realm of M-theory, or more specifically in the properties of string theory in the $A d S_{5} \times S^{5}$ space, it can be applied to most conform-covariant fields.

To understand the basics of this correspondence, recall that the four-dimensional anti-deSitter space-time $A d S_{4}$ can be described as a hypersurface of negative constant curvature isometrically embedded in the flat space $M_{5}(3,2)$. By simply adding one extra space-like dimension, we can easily see that the five-dimensional negative constant curvature surface $A d S_{5}$ can also be seen as a hypersurface embedded in the flat space $M_{6}(4,2)$. Since $A d S_{5}$ is a maximally symmetric sub-space of $M_{6}(4,2)$, it follows that any (pseudo) rotation on that subspace corresponds to a conformal transformation in $M_{4}$, in accordance with the isomorphism

$$
M_{4} \stackrel{\text { conformal }}{\Longleftrightarrow} C_{o} \sim S 0(4,2) \stackrel{\text { isometric }}{\Longrightarrow} A d S_{5}
$$

where it was emphasized that while $C_{o}$ acts conformally on $M_{4}, S O(4,2)$ acts isometrically on $A d S_{5}$. Therefore, for a given conformally invariant field defined in $M_{4}$, there should be a corresponding isometrically invariant field in the gravitational field of the $A d S_{5}$ space.

Example 1: Twistors on the Anti-deSitter space

Since twistors are elements of the spinor representation of the conformal group $C_{o}$ on $M_{4}$, the above isomorphism implies that they can also be seen as the spinor representations of the group $S O(4,2)$, here restricted to be the group of isometries of the $A d S_{5}$ space [9]. Therefore, the $M_{4}$-conformal $/ A d S_{5}$-isometric correspondence defines twistors in the five-dimensional gravitational field of $A d S_{5}$.

Example 2: Gauge Fields on the $A d S_{5}$

The super-symmetric Yang-Mills gauge fields in $A d S_{5}$ can be consistently defined in the heterotic $E_{8} \times E_{8}$ string theory on the 10-dimensional space $A d S_{5} \times S^{5}$. Therefore, using the ADS/CFT correspondence, in the reverse order we may derive a superconformal Yang-Mills field in $M_{4}$, corresponding to the isometric Yang-Mills field in $A d S_{5}$. Reciprocally, the superconformal Yang-Mills field in $M_{4}$ corresponds to a quantum gauge field defined on the gravitational environment of the
$A d S_{5}$, preserving its main quantum properties, including the unitarity.

\section{THE $M_{6}(4,2)$-ISOMETRIC/M $M_{4}$-CONFORMAL CORRESPONDENCE}

The above correspondence should be somehow consistent with four-dimensional physics and not just the $A d S_{5}$. This may be achieved by use of the brane-world theory where, like in the popular Randall-Sundrum model the space $A d S_{5}$ represents the bulk. According to this scheme, all gauge fields are confined to the four-dimensional brane-worlds, but the geometry of these subspaces propagates along the extra dimension. The latter condition requires that all perturbations of the brane-world geometry should belong to the class of fourmanifolds embedded in the same $A d S_{5}$ bulk. In order to see this we require some basic understanding of the geometry of subspaces.

For simplicity we consider here only the case of the 4dimensional brane-world $V_{4}$ embedded in a five dimensional bulk space $V_{5}$, as given by the embedding map $Z^{A}: V_{4} \rightarrow V_{5}$ (Here the indices A,B,C...run from 1 to 5 . The indices $\mu, \nu \ldots$, run from 1 to 4$)$. Together with the unit normal vectors $\eta^{A}$, they define a 5-bein $\left\{z_{, \alpha}^{A}, \eta^{B}\right\}$, in which the components of the bulk's Riemann tensor ${ }^{5} \mathcal{R}_{A B C D}$ can be expressed in terms of the brane-world metric $g_{\mu \nu}$ and the extrinsic curvature $k_{\mu \nu}$ as

$$
\begin{cases}{ }^{5} \mathcal{R}_{A B C D} Z_{, \alpha}^{A} Z_{, \beta}^{B} Z_{, \gamma}^{C} Z_{, \delta}^{D}=R_{\alpha \beta \gamma \delta}-2 k_{\alpha[\gamma} k_{\beta] \delta} & \text { (Gauss) } \\ { }^{5} \mathcal{R}_{A B C D} Z_{, \alpha}^{A} \eta^{B} Z_{, \gamma}^{C} Z_{, \delta}^{D}=k_{\alpha[\gamma ; \delta]} & \text { (Codazzi) }\end{cases}
$$

These expressions become the Gauss-Codazzi equations when the Riemannian metric of the bulk $\mathcal{G}_{A B}$ is given. In the present case this geometry comes from the Einstein-Hilbert principle. Starting from this principle, the dynamics for the embedded brane-world can be derived from the above equations. Indeed, from Gauss' equations we obtain

$$
\begin{array}{r}
{ }^{5} \mathcal{R}_{A B} Z_{, \mu}^{A} Z_{, v}^{B}=R_{\mu \nu} \quad-\left(g^{\alpha \beta} k_{\mu \alpha} k_{v \beta}-h k_{\mu \nu}\right) \\
+{ }^{5} \mathcal{R}_{A B C D} \eta^{A} Z_{, \mu}^{B} Z_{, v}^{C} \eta^{D} \\
{ }^{5} \mathcal{R}=R-\left(K^{2}-h^{2}\right)+2{ }^{5} \mathcal{R}_{A B} \eta^{A} \eta^{B}
\end{array}
$$

where we have denoted $K^{2}=k^{\mu v} k_{\mu v} \quad h=g^{\mu v} k_{\mu v}$. Therefore, the Einstein-Hilbert action for the bulk decomposes into the brane-world geometry as

$$
\begin{aligned}
\int{ }^{5} \mathcal{R} \sqrt{-\mathcal{G}} d^{5} v \equiv \quad & \int\left\{R-\left(K^{2}-h^{2}\right)+2{ }^{5} \mathcal{R}_{A B} \eta^{A} \eta^{B}\right\} \sqrt{-\mathcal{G}} d^{5} v \\
& =\alpha_{*} \int \mathcal{L}^{*} \sqrt{-\mathcal{G}} d^{5} v
\end{aligned}
$$

where we have included the Lagrangian $\mathcal{L}^{*}$ for the confined matter and gauge fields.

Now, in the cases of constant curvature bulks, like in the $A d S_{5}$ case, we have

$$
{ }^{5} \mathcal{R}_{A B C D}=\frac{\Lambda_{*}}{6}\left(\mathcal{G}_{A C} \mathcal{G}_{B D}-\mathcal{G}_{A D} \mathcal{G}_{B C}\right)
$$


where $\Lambda_{*}$ is a bulk cosmological constant. Replacing in the above equations, and applying to a spherically symmetric brane-world, it follows that the extrinsic curvature has a particular form $k_{\mu v}=\alpha_{0} g_{\mu v}$, where $\alpha_{0}$ is an integration constant which cannot be zero, under the penalty of producing just a trivial (a plane) solution $k_{\mu v}=0$. Therefore, the Schwarzschild's solution embedded in $A d S_{5}$ necessarily becomes a Schwarzschild-deSitter space:

$$
d s^{2}=\left(1-\frac{2 m}{r}+\beta_{0}^{2} r^{2}\right)^{-1} d r^{2}+r^{2} d \omega^{2}-\left(1-\frac{2 m}{r}+\beta_{0}^{2} r^{2}\right) d t^{2}
$$

where $\beta_{0}^{2}=\left(3 \alpha_{0}^{2}-\Lambda_{*}\right)$. This shows that not all four-manifolds can be embedded in the $A d S_{5}$ bulk without imposing a constraint in its extrinsic geometry. In particular the Schwarzschild black hole cannot fit into the $A d S_{5}$ bulk.

The above arguments show why the Schwarzschild black holes were not considered in [2]. On the other hand, it is not clear to us that extremal black-holes can be defined and be perturbatively stable in the sense of Nash's theorem [16] within the $A D S_{5}$ bulk. In order to reinstate the Schwarzschild black hole we notice that the spaces $A d S_{5}$ and $M_{6}(4,2)$ have the same 15-parameter group of isometries $S O(4,2)$, such that all arguments based on the symmetries of the $A d S_{5}$, can be extended to $M_{6}(4,2)$. In addition, it can be extended to all four-dimensional isometrically embedded submanifolds of the $M_{6}(4,2)$ bulk and not just those which are embedded in its $A d S_{5}$ hypersurface with this in mind we propose an extension of the ADS/CFT correspondence to this larger class of four-dimensional subspaces of the $M_{6}(4,2)$, provided we may keep a 1:1 correspondence [17]. A local realization of this extension can be obtained by use of the inverse functions theorem, when the embedding functions are also regular. This is precisely the condition which required by Nash's theorem applied to find differentiable solutions of the Gauss-Codazzi equations. The combination of the $M_{6}(4,2)$-isometric/ $M_{4}$ conformal correspondence with the regular and differentiable embeddings provides an extended form of conformal to isometric correspondence, whereby a conformally invariant field in $M_{4}$ corresponds to field on an isometrically and regular embedded brane-world field in $M_{6}(4,2)$. As it happens, $M_{6}(4,2)$ is the regular embedding space for the Schwarzschild solution, so that using such extension the unitarity of the quantum gauge fields near a Schwarzschild black hole may be implemented.
[1] S. Hawking, Comm. Math. Phys. 43, 199 (1975)

[2] S. Hawking, hep-th/0507171, see also J. Baez in www.math.ucr.edu/home/baez/week207

[3] E. Cunningham, Proc. London Math Soc. 8, 77 (1909), H. Bateman, ibid. 8, 223, (1910)

[4] I. Segal, Mathematical Cosmology and Extragalactic Astronomy, Acad. Press (1976)

[5] J. L. Synge, Relativity, The Special Theory (1964)).

[6] B. de Wit \& I. Herger, Anti-deSitter Supersymmetry, hepth/9908005

[7] P. Horava \& E. Witten, Nuc. Phys. B475, 94 (1996)

[8] R. Penrose, J. Math. Phys. 8, 345 (1967)

[9] Y. Murai, Prog. Theor. Phys. 9, 147 (1952), ibid 11, 441 (1954).
[10] L. O'Raifeartaigh, Phys. Rev. Lett. 14, 475 (1965)

[11] S. Coleman \& J. Mandula, Phys. Rev. 159, 1251 (1967).

[12] P. Roman, in Non-compact groups in particle physics, Benjamin (1966).

[13] M. D. Maia Rev. Bras. Fis. (now Brazilian Jour. Phys. ) 11, 429 (1981)

[14] J. Maldacena, Adv. Theor. Math. Phys. 2, 231 (1998)

[15] J. Maldacena, TASI 2003 lectures on ADS/CFT hepth/0309246 v5 (2003)

[16] J. Nash, Ann. Maths. 63, 20, (1956)

[17] M. D. Maia, gr-qc/0505119, to appear in the Intl. Jour. Mod. Phys. D, December (2005). 